\title{
COMPETITION, CONTACTS, AND OTHER FACTORS RESTRICTING NICHES OF PARASITIC HELMINTHS
}

\author{
J. C. HOLMES
}

SUMMARY

Mechanisms that restrict the intraintestinal niches of helminths (as indicated by the sites they occupy) can be favored by several selection pressures. These selection pressures act on different scales, and may interact to have different effects in different circumstances.
In addition, the end results may not reflect responses to the niche, but be a by-product of other processes, such as the immune responses of the host.

\section{RÉsumé : Niches des helminthes parasites : compétition, contacts et autres facteurs limitants.}

Les mécanismes qui limitent la niche des helminthes intestinaux (marquée par leur place dans l'intestin), peuvent être favorisés par diverses pressions sélectives. Ces pressions sélectives agissent à des niveaux différents, et par leurs interactions peuvent, selon les circonstances, avoir des effets différents. En outre, le résultat final peut ne pas refléter seulement les réponses à la niche, mais représenter les effets d'autres processus, comme, par exemple, les réponses immunitaires de l'hôte.
All parasites, like all other organisms, have restricted niches. They are restricted to certain host species, and to certain sites within those hosts. The extent to which niches are restricted, the mechanisms by which those restrictions are effected (proximate causes), the selection pressures leading to the restrictions (ultimate causes), and the effects of those restrictions have all attracted increasing interest over the past decade. A detailed discussion of these topics is clearly impossible in the space available. Instead, I will try to evaluate niche restriction in helminths in the small intestine.

Ecologists distinguish between the "fundamental niche " (the set of conditions required for survival) and the « realized niche " (that portion of the fundamental niche that is actually occupied in a specific system, $i$. e. in an individual host). Realized niches may be only a small part of fundamental niche if populations are small or a species is prevented from using part of the fundamental niche. Because almost all helminths show a clumped distribution amongst host individuals, with most hosts harboring few worms and few harboring large populations, small populations alone should limit realized niches in many cases. Selection pressures may be very different in small and large populations. However, in this paper, I will not focus on population size, but deal with other factors that restrict realized niches.

Department of Zoology, University of Alberta, Edmonton, Alberta, Canada T6G 2E9.
This habitat has been chosen for an important reason. Although a species «niche » (the set of conditions) is distinct from it's " habitat " (its location), the strong physicochemical gradient within the intestine allows a species' position along the intestine to be used as a first approximation of its niche. This approximation does not appear to apply to position within other habitats, such as the gills, where conditions are not so easily represented by position (Kabata, 1959). The analysis of niches (as opposed to microhabitats) in such habitats appears to be much more complex than realized thus far.

I assume that the mechanisms that restrict the intraintestinal niche act within an individual host, and are replicated in other individuals. This assumption treats as random any variation in conditions at any specific site due to individual differences among hosts in diet or physiology, or temporal differences due to seasonal factors or to diel or other feeding regimes. The importance of diel variation (or that associated with feeding regimes) is well shown by the literature on migration of Hymenolepis diminuta and other parasites (e. g., Shostak and Dick, 1989). At present, it is impossible to determine whether a helminth's selection of different sites in different individual hosts reflects selection of the same set of conditions, but in different locations, or that the differences in conditions found in those locations are unimportant to the parasite. It is likely that estimates of the fundamental niche of helminths that are based on the total range occupied in a large set of hosts is an overestimate. Variation amongst host individuals in the conditions present at specific locations in the 
intestinal tract, and the significance of that variation for helminths, appears to be an important topic for investigation.

Given the gradient of conditions along the length of the small intestine, conditions should not be equally favorable throughout the range occupiable by a species. Helminths should select (Crompton, 1976) and preferentially occupy the most favorable parts.

Recent literature suggests three major selective forces for niche restriction in intestinal helminths: specialization, reproductive efficiency, and competition (or required positive interactions). In addition, some authors appear to suggest that niche restrictions are side effects of immune mechanisms, and are not related to any selective forces acting on niches as such.

\section{SPECIALIZATION}

Price (1984) argued that parasites must be highly specialized for specific conditions; part of this specialization may be due to phylogenetic constraints. Changes in conditions along the length of the intestine therefore restrict the sites that can be occupied. There are several lines of evidence that support this concept. First, many helminths do select a restricted portion of the intestine; when introduced into other regions, they move to the preferred area (reviewed by Crompton, 1976). Second, the ranges occupied by gravid specimens are often smaller than those occupied by immature specimens (e. g., Uglem and Beck, 1972), as expected, assuming the requirements for reproduction are more stringent than for survival. Third, when population sizes increase, most helminths do not change their location (as measured by the position of the median individual or unit of biomass), but do extend the range they occupy (for examples, see Stock and Holmes, 1988). For some species, such as the cestode Diorchis ransomi in coots (Pojmanska, 1982) and cestodes of the genus Schistotaenia in grebes (Stock and Holmes, 1988), ranges do not increase much with increasing population size, perhaps the strongest evidence for niche limitation by specialization.

The mechanisms by which niches may be restricted have received little attention. The morphological matching of the scolices of Echeneibothrium and the mucosal surface of specific parts of the spiral intestine of rays are wellknown. The metabolic matching of the aminopeptidases of acanthocephalans and specific parts of the intestine of their host fish (Uglem and Beck, 1972) are less well-known, but such physiological specializations are probably more common.

However, many studies, including those which showed strong evidence for niche restriction by specialization, have shown that other helminths are not so limited in their capabilities, but can live in a high proportion of the small intestine. For example, Stock and Holmes (1988) found that
$3 / 4$ of the species of intestinal helminths in grebes had potential ranges of at least $70 \%$ of the intestinal length, but never occupied that much of the intestine in any individual bird. Realized niches of these species do not appear to be restricted by specialization. The evidence thus far suggests that specialization is important in determining where a species does best (the location of that species in the absence of other constraints), but that most species have wide enough tolerances so that specialization is not a major factor restricting their niches.

\section{REPRODUCTIVE EFFICIENCY}

Rohde (1979) suggested that contact between sexually reproducing helminths that normally live in very small populations should be favored by occupying a limited habitat, to enhance mating opportunities. This hypothesis is supported by at least two lines of evidence. First, mature adult (or gravid) specimens usually have smaller ranges than immature specimens. Second, many intestinal helminths do show marked aggregation of individuals, for example, Echinococcus granulosus in the gut of dogs (Lymbery et al., 1989), and various species of intestinal nematodes (Behnke, 1987). However, these local aggregations may be widely dispersed throughout the intestine. In an increasing number of studies (Haseeb and Fried, 1988), helminths have been shown to release pheromones attractive to other individuals. Concentration of worms by attraction to other worms seems more frequent than concentration by attraction to the same location in the intestine, but more study of the dispersion of helminths in low-intensity infections would be worthwhile.

An extension of the reproductive concentration hypothesis is that segregation of niches among closely related species is due to selection to reinforce reproductive barriers against hybridization (Rohde and Hobbs, 1986). Again, at least two lines of evidence support the hypothesis. First, congeners more often show segregation than related noncongeners (Stock and Holmes, 1988; for an excellent study on gill parasites, see Rohde and Hobbs, 1986). The second line of evidence also comes from Rohde and Hobbs (1986); congeners that occupy overlapping sites have completely different copulatory organs. To my knowledge, no one has investigated the copulatory organs of species of intestinal helminths with overlapping sites. Given the extensive variation in copulatory organs of hymenolepidid cestodes, such a study may provide interesting insights. It seems likely that where closely related species are involved, reinforcement of reproductive isolation is a potent selective force.

\section{COMPETITION}

The selective factor most frequently invoked in analyses of niche occupation is competition. The paradigm is that 
intraspecific competition is a major force extending niche width, with interspecific competition a major force restricting niche overlap (reducing width, producing niche displacement, or both). Although most formal definitions of competition require a limiting resource, most parasitologists use the more general operational definition used by many plant ecologists - a negative interaction between organisms on the same trophic level. Negative interactions can be revealed by reductions in establishment, growth, maturation, or reproduction; displacement of one species is also common (see review in Dobson, 1985).

Dobson (1985) noted that the extent of competition is markedly affected by intensity of the interacting species. With relatively low populations, neither intra- nor interspecific competition are likely to be significant; with relatively high populations, both are likely to be significant. The effects on niche restriction is well-illustrated by Pojmanska's (1982) study of three species of tapeworms (Diorchis spp.) in coots. With low total numbers (1-8) of tapeworms, all three species were found in the anterior ileum; as numbers increased, all three extended their ranges into adjacent regions, but still overlapped extensively. However, at high numbers ( $>250$ worms), the three species occupied smaller ranges in different parts of the gut, with little overlap. This pattern of a shared niche at low densities, but segregated ones at high densities, is consistent with concepts of competition, but not of reproductive isolation.

Mechanisms of competitive niche restriction have not been studied extensively. Direct exploitation of absorbable nutrients seems likely for cestodes and acanthocephalans, which require absorbable monosaccharides (or in some cases, disaccharides) for energy metabolism; these compounds appear to be limiting in the intestine. For example, the ability to use disaccharides such as maltose may allow Moniliformis dubius to displace Hymenolepis diminuta (Keymer et al., 1983). Dobson (1985) gives other examples of exploitation of resources.

Helminths also use interference mechanisms. Crowded $H$. diminuta secrete inhibitors that reduce DNA synthesis in uncrowded worms (Zavras and Roberts, 1985); however, no such inhibitors which act interspecifically have been reported as yet. Several helminths are known to modify their environment in ways adverse to other species (Stock and Holmes, 1987), and a large number of helminths stimulate immune responses more effective against potential competitors (Christensen et al., 1987). Dobson (1985) gives other examples of interference competition, and notes that interference mechanisms are always asymmetric.

\section{NON-ADAPTATIVE RESPONSES}

Local immune or inflammatory responses to intestinal parasites have been interpreted differently. Following such a response, remaining parasites are often limited to other regions of the intestine, suggesting that their niche has been limited by the immune response. Belosevic and Faubert (1983) suggested that the posterior movement of Giardia muris trophozoites during the course of infection was due to an immunological response of the host which made the preferred site uninhabitable. Behnke (1987) suggested that local-scale aggregations of intestinal nematodes may be due to the ability of aggregates, but not individual worms, to modulate immune responses. These examples suggest that the niche restrictions shown in each case may be by-products of the immune response of the host (and counteradaptations by the parasites), and no adaptations in response to any selection pressure on the niche.

Christensen et al. (1987) review a wide variety of cases of antagonistic and synergistic interactions between parasites that they interpret as being due to cross-immune responses, immunologically non-specific responses, or nonspecific parasite-induced immunosuppression. Their examples include many of those covered above. They do not address the question of what selection pressures led to the immune responses, but their interpretation suggests that the niche restrictions that stem from such interactions are also by-products of immune responses, and not adaptive responses to selection pressures.

\section{DISCUSSION}

One clear feature emerging from this review is that different groups of authors interpret the same patterns in very different ways, as the results of different selection pressures (or as the fortuitous outcome of mechanisms selected for totally different functions). It is also apparent that the three major selection pressures are not mutually exclusive, but more likely to reinforce each other, in their effects on niche breadth and overlap. Each mechanism may operate on a variety of spatial scales, although small scale effects may be relatively more important in determining mechanisms of niche restriction in specific systems, if all the possible selective pressures are considered. More investigators should use the careful, unbiased approach of Lymbery et al. (1989).

\section{REFERENCES}

Behnke J. M. : Evasion of immunity by nematode parasites causing chronic infections. Adv. Parasitol., 1987, 26, 2-71.

Belosevic M., Faubert G. M. : Giardia muris: correlation between oral dosage, course of infection, and trophozoite distribution in the mouse small intestine. Exp. Parasitol., 1983, 56, 93-100.

Christensen N. O., Nansen P., Fagbemi B. O., Monrad J. : Heterologous antagonistic and synergistic interactions between helminths and between helminths and protozoans in concurrent experimental infection of mammalian hosts. Parasitol. Res., 1987, $73,387-410$. 


\section{J. C. HOLMES}

Crompton D. W. T. :. Entry into the host and site selection. In: Ecological aspects of parasitology, Kennedy C. R. (ed.). NorthHolland Publ. Co., Amsterdam, 1976, 41-73.

Dobson A. P. : The population dynamics of competition between parasites. Parasitology, 1985, 91, 317-347.

Haseeb M. A., Fried B. : Chemical communication in helminths. Adv. Parasitol., 1988, 27, 170-207.

Kabata Z. : Ecology of the genus Acanthochondria Oakley (Copepoda Parasitica). J. Marine Biol. Assoc. United Kingdom, 1959, 38, 249-261.

Keymer A. E., Crompton D. W. T., Singhvi A. : Mannose and the "crowding effect" of Hymenolepis in rats. Int. J. Parasitol., 1983, 13, 561-570.

Lymbery A. J., Hobbs R. P., Thompson R. C. A. : The dispersion of Echinococcus granulosus in the intestine of dogs. $J$. Parasitol., 1989, 75, 562-570.

Pojmanska T. : The co-occurrence of three species of Diorchis Clerc, 1903 (Cestoda: Hymenolepididae) in the European coot, Fulica atra L. Parasitology, 1982, 84, 419-429.

Price P. W. : Evolutionary biology of parasites. Princeton University Press, Princeton, N. J., 1980.

Rohde K. : A critical evaluation of intrinsic and extrinsic factors responsible for niche restriction in parasites. Amer. Natural., 1979, 114, 648-671.

Rohde K., Hobbs R. P. : Species segregation: competition or reinforcement of reproductive barriers? In: Parasite lives, Cremin C., Dobson C., Moorehouse D. E. (eds). University of Queensland Press, St. Lucia, Queensland, 1986, 189-199.

Shostak A. W., Dick T. A. : Helminth position within the intestine of naturally-infected pike (Esox lucius) relative to host stomach contents. J. Parasitol., 1989, 75, 905-910.

Stock T. M., Holmes J. C. : Dioecocestus asper (Cestoda: Dioecocestidae): an interference competitor in an enteric helminth community. J. Parasitol., 1987, 73, 1116-1123.

Stock T. M., Holmes J. C. : Functional relationships and microhabitat distributions of enteric helminths of grebes (Podicipedidae): the evidence for interactive communities. J. Parasitol., 1988, 74, 214-227.

Uglem G. L., Beck S. M. : Habitat specificity and correlated aminopeptidase activity in the acanthocephalans Neoechinorhynchus cristatus and N. crassus. J. Parasitol., 1972, 58, 911-920.

Zavras E. T., Roberts L. S. : Developmental physiology of cestodes: cyclic nucleotides and the identity of putative crowding factors in Hymenolepis diminuta. J. Parasitol., 1985, 71, 96-105. 